Supplementary Information for

\title{
Macrocyclization of a Class of Camptothecin Analogues into Tubular Supramolecular Polymers
}

Hao $\mathrm{Su}^{1}$, Feihu Wang ${ }^{1}$, Yuzhu Wang ${ }^{1}$, Andrew G. Cheetham ${ }^{1}$, and Honggang Cui ${ }^{1,2,3 *}$.

${ }^{1}$ Department of Chemical and Biomolecular Engineering, and Institute for

NanoBioTechnology, The Johns Hopkins University, 3400 North Charles Street,

Baltimore, Maryland 21218, United States.

${ }^{2}$ Center for Nanomedicine, The Wilmer Eye Institute, Johns Hopkins University School of Medicine, 400 North Broadway, Baltimore, Maryland 21231, United States.

${ }^{3}$ Department of Oncology and Sidney Kimmel Comprehensive Cancer Center, The Johns Hopkins University School of Medicine, Baltimore, Maryland 21205, United States.

This PDF file includes: materials and methods, experimental protocols and other supplementary text, supplementary Fig. S1-S12, supplementary Table S1 and supplementary Scheme S1-S3.

\section{S1 Materials and Methods}

Fmoc amino acids, HBTU and Rink amide MBHA resin were sourced from Advanced Automated Peptide Protein Technologies (AAPPTEC, Louisville, KY, USA). mPEG $4-\mathrm{CH}_{2} \mathrm{CH}_{2} \mathrm{COOH}\left(\mathrm{OEG}_{5}-\mathrm{COOH}\right)$ was purchased from ChemPep Inc. (Wellington, FL, USA). Camptothecin was purchased from Chem-Impex International Inc. (Wood Dale, IL, USA) and all other reagents were sourced from Sigma-Aldrich (St. Louis, MO) or VWR (Radnor, PA, USA), unless otherwise stated.

RP-HPLC was performed on a Varian ProStar Model 325 HPLC (Agilent Technologies, Santa Clara, CA). Preparative separations utilized a Varian PLRP-S column $(100 \AA, 10 \mu \mathrm{m}, 150 \times 25 \mathrm{~mm})$, while analytical HPLC used a Varian Pursuit XRs C18 column $(5 \mu \mathrm{m}, 150 \times 4.6 \mathrm{~mm})$. Water and acetonitrile containing $0.1 \% \mathrm{v} / \mathrm{v}$ TFA were used as the mobile phase. Purified fractions were lyophilized using a FreeZone $-105{ }^{\circ} \mathrm{C} 4.5 \mathrm{~L}$ freeze dryer (Labconco, Kansas City, MO). ESI-MS mass spectrometric data was acquired on a Finnigan LDQ Deca ion-trap mass spectrometer (Thermo-Finnigan, Waltham, MA).

\section{S2 Supplementary Text}

S2.1 Synthesis of Tubustecans (TTs)

All peptide sequences were synthesized on Rink Amide MBHA resins using standard 9-fluorenylmethoxycarbonyl (Fmoc) solid phase synthesis techniques on a $0.25 \mathrm{mmol}$ scale. Scheme S1A illustrates the manual synthetic protocols used for synthesizing peptidic precursors. The three peptides dCys- $\mathrm{K}_{2}$, dCys- $\mathrm{E}_{2}$, and $\mathrm{dCys}-\mathrm{KE}$ used similar synthetic procedures by sequentially adding amino acids. 
Fmoc-Lys(Fmoc)-OH was introduced as a branching motif to yield a dual-functional reaction point. Following Fmoc removal, Fmoc-Cys(Trt)-OH was conjugated onto each $\mathrm{N}$-terminus to furnish thiol groups for drug conjugation. All Fmoc deprotections were performed using a $20 \%$ 4-methylpiperidine in DMF solution for 15 min and repeated once. The amino acid coupling was performed after Fmoc deprotection by adding a mixture of Fmoc-amino acids, HBTU and DIEA (4:4:6 molar equiv to resin) in DMF for $2 \mathrm{~h}$. Acetylation was performed on the $\alpha$-amino groups of $N$-termini amino acids using a $20 \%$ acetic anhydride in DMF solution with $100 \mu \mathrm{L}$ DIEA, shaking for $15 \mathrm{~min}$ and repeating twice. After finishing the whole sequence, peptides were cleaved from the resin support using a mixture of TFA/TIS/ $\mathrm{H}_{2} \mathrm{O}$ at a ratio of 95:2.5:2.5 for $3 \mathrm{~h}$. Excess TFA was removed by rotary evaporation and the concentrated solution was precipitated in cold diethyl ether to obtain the crude peptides, which were collected and dried under vacuum overnight.

In the synthesis of dCys-OEG2, two Fmoc-Lys(Mtt)-OH molecules were first loaded onto the resin to allow selective deprotection and functionalization of the lysine side chain amino groups. Following Mtt deprotection (3\% TFA, 5\% TIS, 92\% DCM), $\mathrm{OEG}_{5}-\mathrm{COOH}$ was conjugated onto the side chain of the lysine through amide bond formation in the manner described earlier for the amino acid couplings (Scheme S1A).

The synthesis of functional TTs was carried out by mixing CPT-etcSS-Pyr and the corresponding crude peptides synthesized above in $\mathrm{N}_{2}$-purged DMSO with a molar ratio of 3:1 (Scheme S1B). After reacting for 2 days, the mixture was diluted with 0.1\% TFA in acetonitrile/water and purified by preparative RP-HPLC. All separations were performed using a flow rate of $20 \mathrm{~mL} / \mathrm{min}$ for 25 mins in total, monitoring at $362 \mathrm{~nm}$. The mobile phase gradient began at $15 \% \mathrm{MeCN}$, increasing to $80 \% \mathrm{MeCN}$ over $20 \mathrm{~min}$, and then holding for $2 \mathrm{~min}$ before returning to initial conditions over $3 \mathrm{~min}$. Collected fractions were analyzed by ESI-MS (Fig. S1-S4) and the appropriate fractions were combined, concentrated, and lyophilized on a FreeZone $-105{ }^{\circ} \mathrm{C} 4.5 \mathrm{~L}$ freeze dryer. The powders obtained were then re-dissolved, calibrated, and aliquotted into cryo-vials before re-lyophilization.

The purity of the conjugates was proven by analytical RP-HPLC using the following conditions: the flow rate was $1 \mathrm{~mL} / \mathrm{min}$, with the mobile phase gradient starting from $5 \%$ $\mathrm{MeCN}$ (with $0.1 \% \mathrm{TFA}$ ), increasing to $95 \% \mathrm{MeCN}$ (with $0.1 \% \mathrm{TFA}$ ) over $15 \mathrm{~min}$, and then holding for $1 \mathrm{~min}$ before returning to the initial conditions over $4 \mathrm{~min}$; the monitored wavelength was $362 \mathrm{~nm}$ (Fig. S1-S4). Molecular masses were determined using ESI-MS (Fig. S1-S4).

\section{S2.2 Calibration of the TT concentration}

The concentrations of purified TTs were determined by calculating the amount of free CPT produced from the prodrugs upon reduction of the disulfide linker. $25 \mu \mathrm{L}$ stock solution of the corresponding prodrug in $\mathrm{MeCN} / \mathrm{H}_{2} \mathrm{O}(1: 1)$ was diluted to $50 \mu \mathrm{L}$ by adding $25 \mu \mathrm{L} 1 \mathrm{M}$ TCEP solution in $\mathrm{MeCN} / \mathrm{H}_{2} \mathrm{O}$ (1:1) and mixing via periodic vortexing. $25 \mu \mathrm{L}$ of the solution was then injected onto the HPLC (so as to completely fill the $20 \mu \mathrm{L}$ loop), measuring the area under the peak of free CPT at $362 \mathrm{~nm}$. The CPT concentration of treated conjugates was obtained by comparison with the standard calibration curve of CPT. The TT concentration was calculated based on the applied dilutions and number of 
CPT molecules. Finally, the stock solution was diluted to $200 \mu \mathrm{M}, 400 \mu \mathrm{M}, 1 \mathrm{mM}$ and 5 $\mathrm{mM}$ according to the calibrated concentration and aliquotted into cryo-vials before re-lyophilization.

CPT Standard calibration curve: $y=17.095 x+59.358$, where $y$ is the area under the cruve and $x$ is the concentration of CPT $(\mu \mathrm{M})$.

\section{S2.3 Transmission electron microscopy (TEM) protocol}

The self-assembly of TTs was promoted by directly dissolving TTs into deionized water at concentrations of $200 \mu \mathrm{M}, 400 \mu \mathrm{M}$ and $800 \mu \mathrm{M}$. The solutions were all tuned to neutral $\mathrm{pH}$ by the addition of trace amount of $1 \mathrm{M} \mathrm{NaOH}$ or $\mathrm{HCl}$, sonicated for $5 \mathrm{~min}$ in $50{ }^{\circ} \mathrm{C}$ water bath and then aged overnight at room temperature. TEM samples were prepared by depositing $7 \mu \mathrm{L}$ of the appropriate solution onto a carbon-coated copper grid (Electron Microscopy Services, Hatfield, PA, USA), wicking away the excess solution with a small piece of filter paper. Next, $7 \mu \mathrm{L}$ of a $2 \mathrm{wt} \%$ uranyl acetate aqueous solution was deposited on the surface for 30 seconds, wicking away the excess solution with filter paper. The grids were then air-dried overnight at room temperature prior to imaging. Bright-field TEM imaging was performed using an FEI Tecnai 12 TWIN Transmission Electron Microscope operated at an acceleration voltage of $100 \mathrm{kV}$. All TEM images were acquired by a SIS Megaview III wide-angle CCD camera or 16 bit $2 \mathrm{~K} \times 2 \mathrm{~K}$ FEI Eagle bottom mount camera.

\section{S2.4 Cryogenic transmission electron microscopy (Cryo-TEM) protocol}

Cryo-TEM imaging was performed using higher sample concentrations of $800 \mu \mathrm{M}$ (compared with $200 \mu \mathrm{M}$ for conventional TEM imaging). Extended imaging times can result in damage to the vitreous ice film caused by the electron beam and so higher concentrations can allow a more rapid visualization that reduces this likelihood. $6 \mu \mathrm{L}$ of the appropriate solution was dropped onto a lacey carbon-film-supported TEM copper grid (Electron Microscopy Services, Hatfield, PA, USA). All the TEM grids used for cryo-TEM imaging were pretreated with plasma air to render the lacey carbon film hydrophilic. A thin film of the sample solution was produced using a Vitrobot with a controlled humidity chamber (FEI). After loading of the sample solution, the lacey carbon grid was blotted using preset parameters and plunged instantly into a liquid ethane reservoir precooled by liquid nitrogen. The vitrified samples were then transferred to a cryo-holder and cryo-transfer stage that was cooled by liquid nitrogen. To prevent sublimation of vitreous water, the cryo-holder temperature was maintained below $-170{ }^{\circ} \mathrm{C}$ during the imaging process. All images were recorded by a 16 bit $2 \mathrm{~K} \times$ 2K FEI Eagle bottom mount camera.

S2.5 Circular dichroism (CD) spectroscopy of TT 1 tubular supramolecular polymers (SPs)

All CD spectra were recorded on a Jasco J-710 spectropolarimeter (JASCO, Easton, MD, USA) from 200 to $480 \mathrm{~nm}$ using $1 \mathrm{~mm}$ path length quartz UV-Vis absorption cell (Thermo Fisher Scientific, Pittsburgh, PA, USA). TT 1 solution of $200 \mu \mathrm{M}$ was

measured and the obtained spectrum was converted from ellipticity (mdeg) to molar 
ellipticity $\left(\mathrm{deg} \cdot \mathrm{cm}^{2} \cdot \mathrm{dmol}^{-1}\right)$. The background spectrum of the solvent was acquired and subtracted from the sample spectrum.

S2.6 CD measurements of TT solutions

Various TT solutions of $200 \mu \mathrm{M}$ were measured according to the protocols described in section 2.5. Collected data was converted from ellipticity (mdeg) to molar ellipticity (deg. $\mathrm{cm}^{2} \cdot \mathrm{dmol}^{-1}$ ) and is shown in Fig. S6. CD spectra for all four TT molecules were further normalized by the maximum intensity to verify the similarity of their CD spectra (Fig. 3A).

S2.7 Critical micellization concentration (CMC) measurement of TTs via Nile Red encapsulation

Nile Red is a hydrophobic, solvatochromic dye that fluoresces intensely upon exposure to hydrophobic environments compared with its strongly quenched and red-shifted fluorescence in aqueous environments. The CMC of the TTs was determined by incubating these molecules at various concentrations with a fixed content of Nile Red. $10 \mu \mathrm{L}$ of a $1 \mathrm{mM}$ Nile Red stock solution in acetone was added to each microcentrifuge tube to be used, with the acetone allowed to evaporate in a dark area. $1 \mathrm{~mL}$ TT solutions of various concentrations were subsequently added to the Nile Red containing tubes and equilibrated overnight. The emission spectrum for each sample was then recorded on a Fluorolog spectrofluorometer (Horiba Jobin Yvon Inc., Edison, NJ), acquiring between 580 and $720 \mathrm{~nm}$ with an excitation wavelength of $550 \mathrm{~nm}$. The ratio of intensity at $635 \mathrm{~nm}$ (emission maximum of the dye in hydrophobic environment) to that at $660 \mathrm{~nm}$ (emission maximum in aqueous conditions) was then plotted against the concentration of each TT, which shows a transition in the data when the TT concentration exceeded the CMC (Fig. S7).

\section{S2.8 Zeta potential measurement}

TT solutions of $200 \mu \mathrm{M}$ in 1X-DPBS buffer $(\mathrm{pH}=7.4)$ were prepared by directly mixing identical volumes of a $400 \mu \mathrm{M}$ aqueous TT solution and $2 \mathrm{X}$-DPBS buffer $(\mathrm{pH}=$ 7.4). TT solutions of $200 \mu \mathrm{M}$ in $1 \mathrm{X}$-DPBS buffer $(\mathrm{pH}=5.0)$ were prepared by mixing identical volumes of a $400 \mu \mathrm{M}$ aqueous TT solution and 2X-DPBS buffer $(\mathrm{pH}=5.0)$, which was pretreated with $6 \mathrm{M} \mathrm{HCl}$. The zeta potential measurements were performed on a Zetasizer Nano ZS90 (Malvern Instruments Ltd., UK). The prepared solutions were loaded in capillary cells and equilibrated for $2 \mathrm{~min}$ prior to measurement. The average values and their standard deviations are calculated from three replicate measurements. The zeta potential of the assembled structures was obtained by measuring the electrophoretic movement of the nanostructures under the applied electric field, where the movement velocity is determined by phase analysis light scattering. Variations of the zeta potential value over time were determined by measuring the solutions at different aging time points (1, 2, 4 and 7 days) and are plotted in Fig. 3C and Fig. S8.

S2.9 Drug release from TT 1 tubular supramolecular polymers

The release of free CPT from TT 1 SPs was evaluated in the presence or absence of GSH. $400 \mu \mathrm{M}$ stock solutions of TT 1 in deionized water were prepared and diluted to 
$200 \mu \mathrm{M}$ with $20 \mathrm{mM}$ PBS buffer with or without GSH $(20 \mathrm{mM})$. Three replicate experiments were prepared together for both conditions. The solutions were incubated at $37^{\circ} \mathrm{C}$ and samples were collected at $0 \mathrm{~min}, 10 \mathrm{~min}, 20 \mathrm{~min}, 30 \mathrm{~min}, 1 \mathrm{~h}, 2 \mathrm{~h}, 4 \mathrm{~h}, 6 \mathrm{~h}, 8 \mathrm{~h}$, $12 \mathrm{~h}$ and $24 \mathrm{~h}$. For each collected sample, the reductive release was halted by acidification of the solution through the addition of $0.2 \mu \mathrm{L}$ of $2 \mathrm{M} \mathrm{HCl}$. Samples were then frozen with liquid nitrogen and stored at $-30{ }^{\circ} \mathrm{C}$ until analysis. The amount of released CPT was monitored by RP-HPLC using the following conditions: Varian Pursuit XRs $\mathrm{C}_{18}(5 \mu \mathrm{m}, 150 \times 4.6 \mathrm{~mm}) ; 362 \mathrm{~nm}$ detection wavelength; $1 \mathrm{ml} / \mathrm{min}$ flow rate; the gradient began at $90 \%$ of mobile phase A ( $0.1 \%$ aqueous TFA) and $10 \%$ of mobile phase $\mathrm{B}$ (acetonitrile containing $0.1 \%$ TFA) increasing to $90 \%$ mobile phase B by $15 \mathrm{~min}$ and held for another $1 \mathrm{~min}$ before decreasing to the initial solvent composition at 20 minutes. Selected time points were characterized and data were plotted as a percentage of the total expected CPT concentration. It was found that $80 \%$ of the conjugated CPT molecules were released within $2 \mathrm{~h}$ in the presence of GSH, reaching almost $100 \%$ by $6 \mathrm{~h}$, while only a slight amount (less than 10\%) of the conjugates had degraded in $24 \mathrm{~h}$ without GSH (Fig. S9). Detailed HPLC traces at different time points were also summarized, clearly demonstrating the release trend of free CPT (Fig. 4A).

\section{S2.10 Physical stability of non-ionic TT 1 supramolecular polymer}

The stability of non-ionic TT 1 SPs upon dilution in cell medium was evaluated by recording the $\mathrm{CD}$ spectrum for a series of prepared dilutions. Phenol red-free DMEM (Mediatech) containing 10\% fetal bovine serum (FBS, Invitrogen) and 1\% antibiotics (Invitrogen) was used as the cell medium solution, thereby avoiding any potential spectroscopic interferences that would otherwise be caused by the dye. A stock solution of TT 1 was prepared at a concentration of $1 \mathrm{mM}$ in water and aged overnight. The aged solution was then diluted to $200 \mu \mathrm{M}$ with cell medium. Further dilutions of the $200 \mu \mathrm{M}$ TT 1 in cell medium were prepared at $100 \mu \mathrm{M}, 50 \mu \mathrm{M}, 25 \mu \mathrm{M}, 10 \mu \mathrm{M}$, and $5 \mu \mathrm{M}$ concentration. All diluted solutions were incubated for an additional two days before CD measurements were made. All the CD spectra were recorded from 300 to $440 \mathrm{~nm}$ using a $1 \mathrm{~mm}$ (for $200 \mu \mathrm{M}, 100 \mu \mathrm{M}$, and $50 \mu \mathrm{M}$ ) or $10 \mathrm{~mm}$ (for $25 \mu \mathrm{M}, 10 \mu \mathrm{M}$, and $5 \mu \mathrm{M}$ ) path length quartz cell. The spectra were collected and normalized from ellipticity (mdeg) to molar ellipticity $\left(\mathrm{deg} \cdot \mathrm{cm}^{2} \cdot \mathrm{dmol}^{-1}\right)$. No significant changes were observed in the normalized curves, indicating no obvious disassociation of the self-assembled SPs structures at concentrations above $25 \mu \mathrm{M}$ (Fig. 4B). Further dilution of TT 1 solution to 10 and $5 \mu \mathrm{M}$ resulted in a slight decrease of CD signal at $389 \mathrm{~nm}$, suggesting that higher monomer/nanostructure ratio and likely partial dissociation of nanostructures as the concentration approaches the CMC. In addition, the inset photograph (Fig. 4B) shows that a solution of TT 1 in cell medium remains clear after a week, suggesting no obvious aggregation of SPs in cell medium and long-term stability.

\section{S2.11 Cytotoxicity studies of TTs against U87 MG brain tumor cell.}

The human brain cancer cell line U87 MG was a generous gift from Dr. Wirtz (ChemBE, JHU). DMEM (Invitrogen) containing 10\% fetal bovine serum (FBS, Invitrogen) and 1\% antibiotics (Invitrogen) was used for the culture of the U87 MG cells. Cancer cells were incubated at $37^{\circ} \mathrm{C}$ in a humidified incubator (Oasis, Caron, Marietta, 
$\mathrm{OH}, \mathrm{USA}$ ) with an atmosphere of $5 \% \mathrm{CO}_{2}$. The cytotoxicities of TTs were evaluated using the SRB method. U87 MG cells were seeded onto 96-well plates (5000 cells/well) and allowed to attach overnight. $1 \mathrm{mM}$ aqueous stock solutions of TTs were prepared and aged overnight. The stock solutions were then diluted with fresh medium to achieve final CPT concentrations of $0.1,1,10,100,500,1000,5000$ and $10000 \mathrm{nM}$. After dilution, the TT-containing media were used to incubate cells immediately. Medium containing the same concentration of free CPT ranging from 0.1 to $10000 \mathrm{nM}$ was also used to incubate the cells, with non-treated cells (solvent only) as the control group. In addition, irinotecan at the concentrations of $0.1,1,5,10,50,100$ and $500 \mu \mathrm{M}$ was employed as a second control. After $48 \mathrm{~h}$ incubation, the cell viability was evaluated using the SRB method according to the manufacturer's protocols (TOX-6, Sigma, St. Louis, MO). The results suggest that TTs showed an enhanced efficacy against U87 MG cells compared with irinotecan and were even comparable to its parent drug CPT.

\section{S2.12 In vivo animal studies}

All animal experiments conducted with mice were performed at the Johns Hopkins University (JHU) in accordance with protocols approved by the JHU Institutional Animal Care and Use Committee (IACUC). Female athymic nude mice were obtained from the Charles River and kept at the JHU Animal Care Facility. The animals were acclimatized to the laboratory environment for at least one week prior to the experiments.

\section{S2.13 Maximum tolerated dose (MTD) determination}

The MTD was determined using healthy female athymic nude mice (Charles River, 12-13 weeks old). A single dose of TT 1 was administered through intravenous injection on day 1 and the body weights of each mouse were recorded every day from day 1 to day $16(\mathrm{n}=3)$. The dosing volume was determined based upon a ratio of $200 \mu \mathrm{L}$ for a $20 \mathrm{~g}$ mouse and was scaled appropriately according to the actual body weight of the mice. Doses were 54, 36, 30, 27, 24, 21, 18, 15, 12, 9 and $4.5 \mathrm{mg} / \mathrm{kg}$ (CPT equivalent) (Fig. S11). The maximum tolerated dose (MTD) was determined by the largest dose that did not result in more than a $20 \%$ mean body weight loss or death of an animal in that group. Doses of 54 and $36 \mathrm{mg} / \mathrm{kg}$ caused at least one death in each group.

\section{S2.14 Antitumor efficacy study for systemic delivery}

A total of $2 \times 10^{6}$ human glioblastoma U87 MG cells were subcutaneously injected into the right shoulder of athymic nude mice (8-9 weeks old). The mice were used for the efficacy study after three to four weeks when the tumor had reached about 190-250 $\mathrm{mm}^{3}$ in size. Mice were randomly divided into six groups $(n=5)$ of non-treated, CPT (4.5 $\mathrm{mg} / \mathrm{kg})$, Irinotecan $(60 \mathrm{mg} / \mathrm{kg}), 4.5 \mathrm{mg} / \mathrm{kg}, 9 \mathrm{mg} / \mathrm{kg}$ and $15 \mathrm{mg} / \mathrm{kg}$ TT 1 . Water insoluble free CPT was dissolved/suspended in a mixture of DMSO/ethanol/PEG-400/water $(1: 1: 2: 1)$. CPT $(4.5 \mathrm{mg} / \mathrm{kg})$ and irinotecan $(60 \mathrm{mg} / \mathrm{kg})$ were administered by intraperitoneal injection, while freshly prepared TT 1 solutions of various doses were administered intravenously by tail vein injection every 4 days for a total of three doses (days 1, 5, and 9). The dosing volume of TTs and irinotecan was determined based upon a ratio of $200 \mu \mathrm{L}$ for a $20 \mathrm{~g}$ mouse and was scaled appropriately according to the actual body weight of the mice. The dosing volume of CPT was determined based upon a ratio 
of $100 \mu \mathrm{L}$ for a $20 \mathrm{~g}$ mouse and was scaled appropriately according to the actual body weight of the mice. Tumor volumes were measured and recorded every other day. The body weights were measured and recorded every day or every other day. The tumor volume was determined by measuring the tumor in two dimensions with calipers and using the formula "tumor volume $=\left(\right.$ length $\left.\times w_{i d t h}^{2}\right) / 2$ ". Each animal was euthanized once the tumor volume reached the predetermined end point size of $2000 \mathrm{~mm}^{3}$.

\section{S2.15 Encapsulation of functional dye molecules}

A $300 \mu \mathrm{M}$ in water stock solution of TT 1 was prepared and aged overnight to form tubular SPs. Hydrophobic dyes, such as Coumarin 6 (C6), Nile Red, Rose Bengal lactone (Rose), and IR 780 iodide (IR 780), were dissolved in acetonitrile at a concentration of $600 \mu \mathrm{M}$ (Scheme S3). To perform the dye encapsulation experiments, $400 \mu \mathrm{L}$ of the pre-formed TT 1 solution and $200 \mu \mathrm{L}$ of the corresponding dye solution in acetonitrile were mixed together to make a final solution of $600 \mu \mathrm{L}\left(\mathrm{H}_{2} \mathrm{O} / \mathrm{MeCN}=2: 1\right.$, v/v) in which the concentration of both prodrug and dye was $200 \mu \mathrm{M}$ (prodrug/dye $=1: 1, \mathrm{~mol} / \mathrm{mol}$ ). The resulting mixed TT 1 and dye solution was aged overnight to allow the penetration of dye molecules into the tubular structures and then directly lyophilized to fully remove all the solvents. Next, the lyophilized powder was reconstituted to a final prodrug concentration of $200 \mu \mathrm{M}$ by the addition of $600 \mu \mathrm{L}$ of water, vortexing for $60 \mathrm{~s}$, and sonicating for $5 \mathrm{~min}$ in $50{ }^{\circ} \mathrm{C}$ water bath. The solutions were aged for at least $6 \mathrm{~h}$ before being centrifuged (6000 rpm, $5 \mathrm{~min})$ to remove any precipitated free dye. The supernatant was collected for further study. The dye loading capacity was calculated from the percentage of dye in the supernatant to the sum of encapsulated dye and added prodrugs. To get a higher resolution of dye-doped TT 1 solution, the solutions imaged in Figure 5A were concentrated to a concentration of $800 \mu \mathrm{M}$. 
S3 Additional Figures, Tables and Schemes.
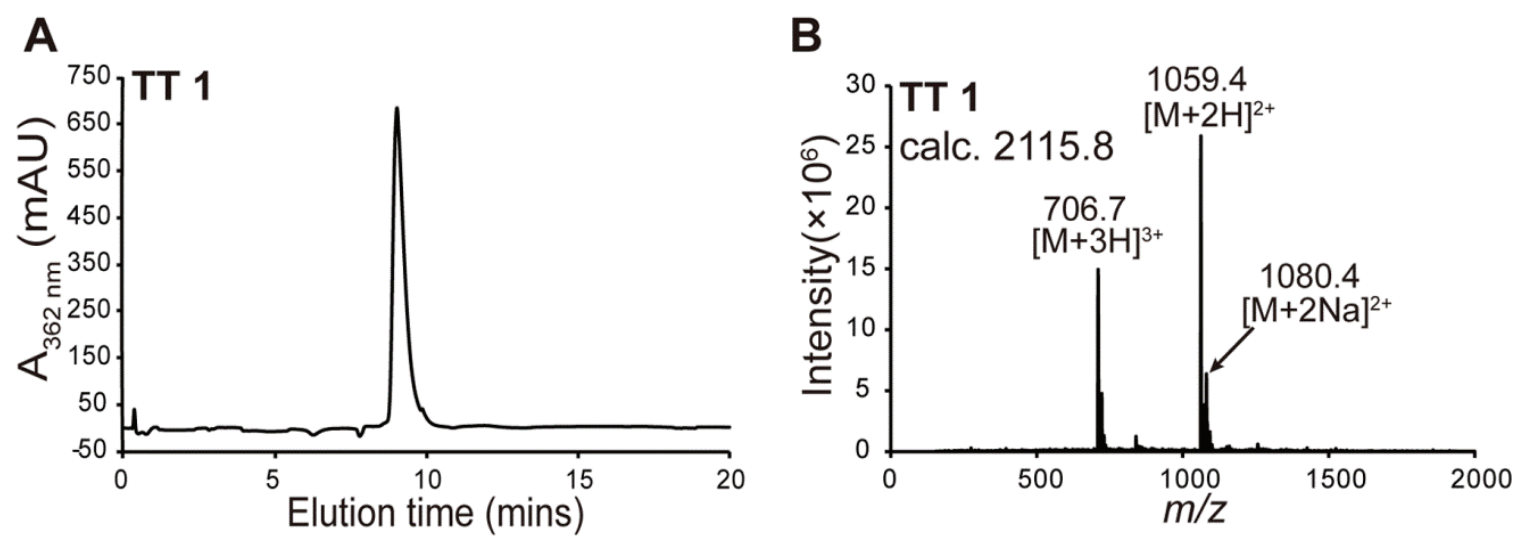

Fig. S1. RP-HPLC chromatogram (A) and ESI-MS spectrum (B) of TT 1.

A

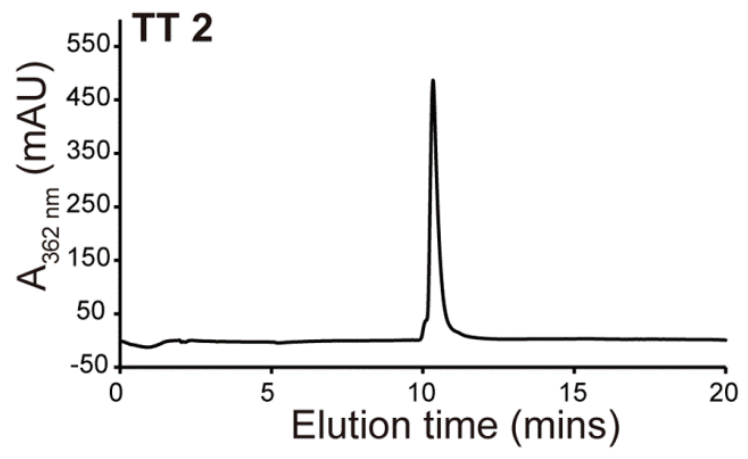

B

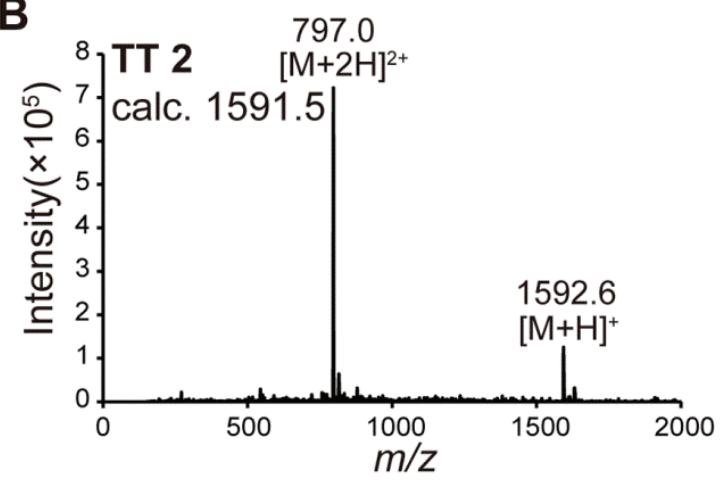

Fig. S2. RP-HPLC chromatogram (A) and ESI-MS spectrum (B) of TT 2.

A

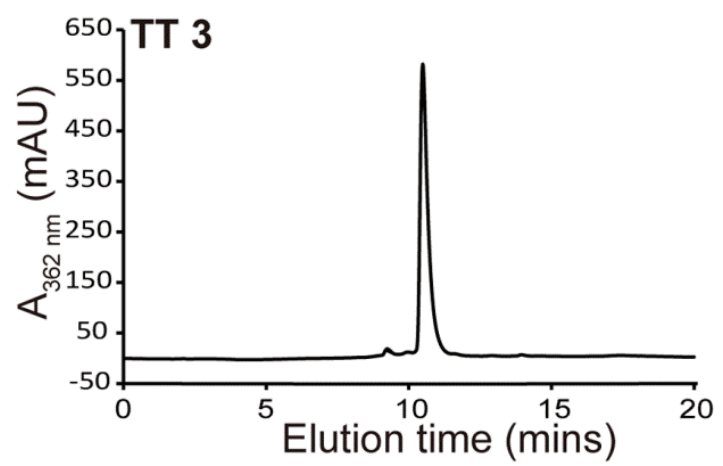

B

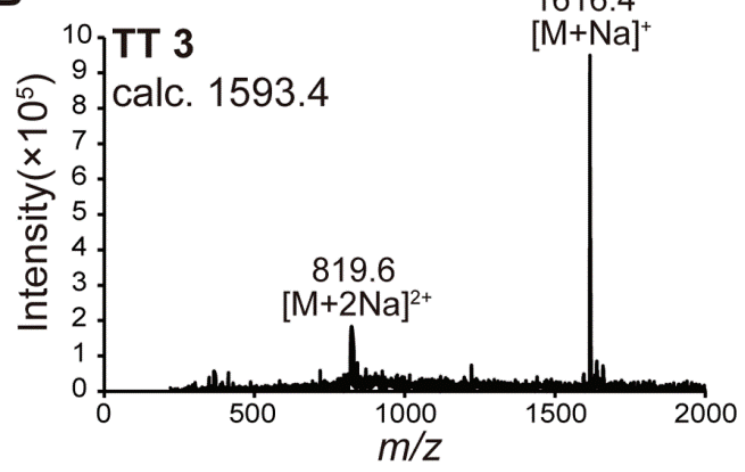

Fig. S3. RP-HPLC chromatogram (A) and ESI-MS spectrum (B) of TT 3. 
A

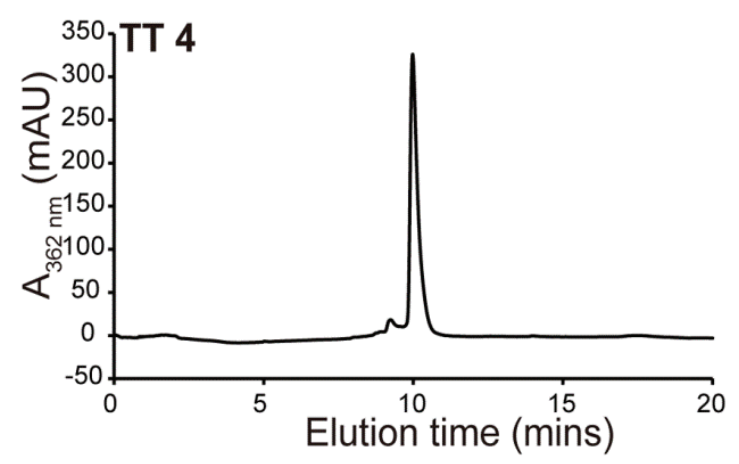

B

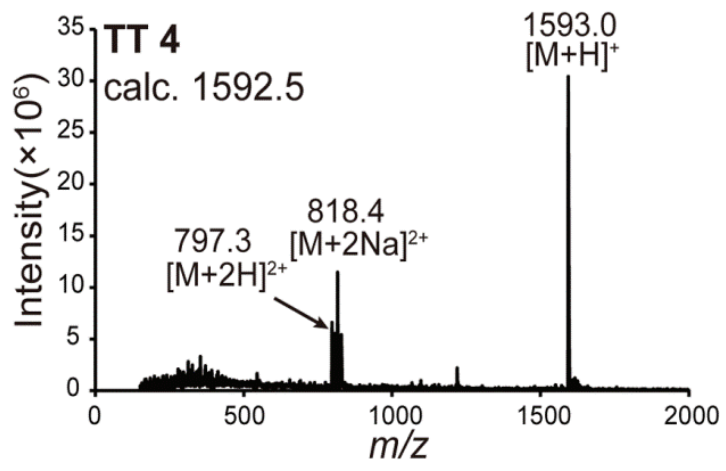

Fig. S4. RP-HPLC chromatogram (A) and ESI-MS spectrum (B) of TT 4.

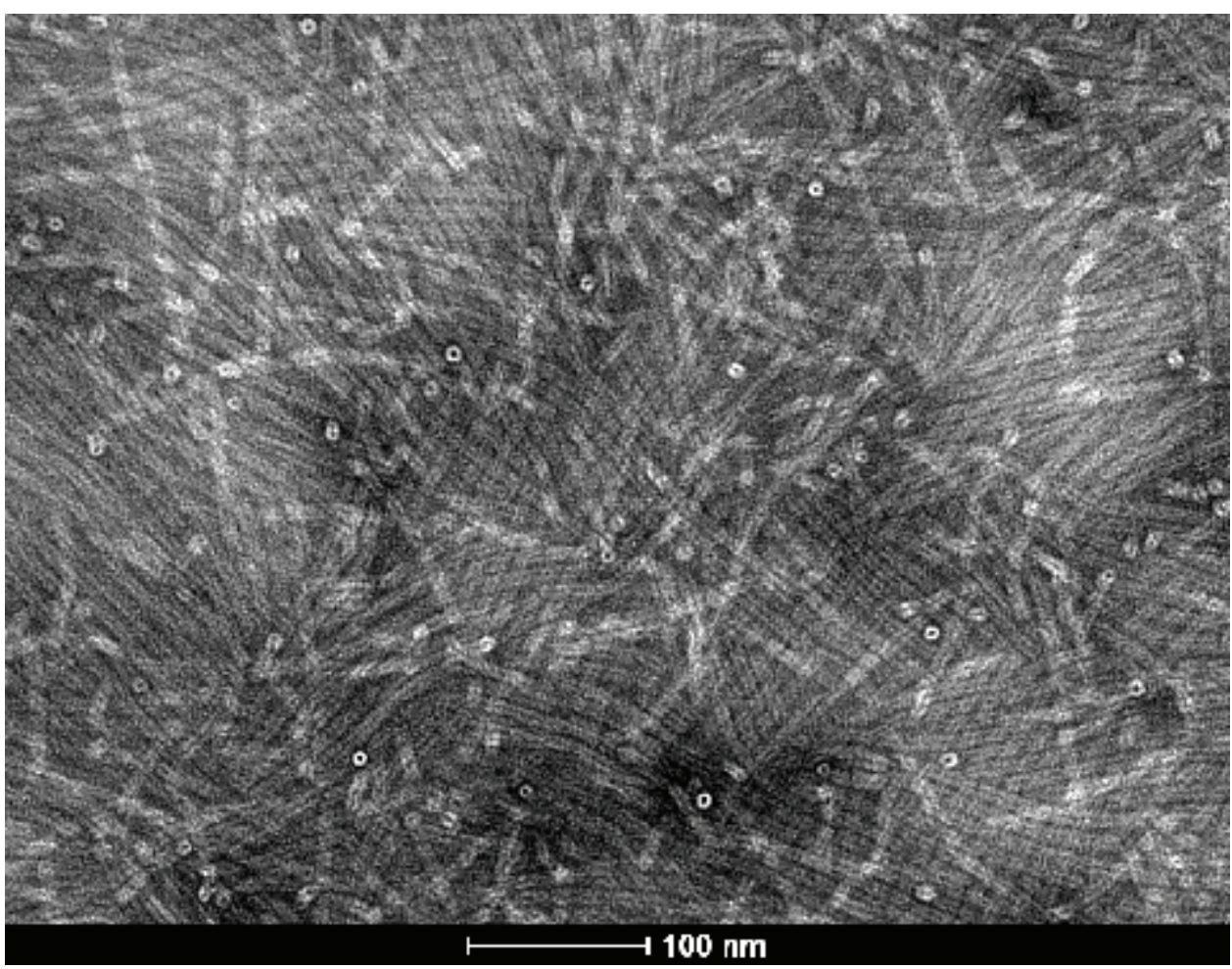

Fig. S5. TEM image of self-assembled TT 1 that illustrates the occasional presence of toroidal structures. The wall thickness and diameter of inner cavities of TT 1 tubular SPs were determined by measuring these observed toroids $(n>50)$. 


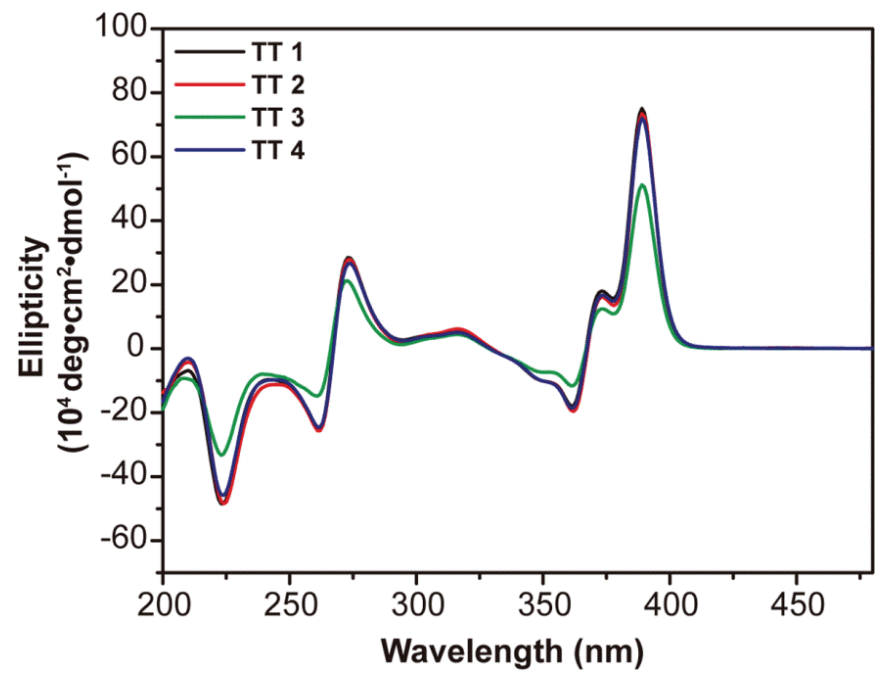

Fig. S6. CD spectra of different TT tubular SPs in aqueous solution at $\mathrm{pH}=7.4$. After normalizing by maximum intensity (Fig. 4A), the CD spectra for all four TTs showed similar pattern, further confirming the semblable tubular morphology of four nanostructures. The concentrations are $200 \mu \mathrm{M}$. 

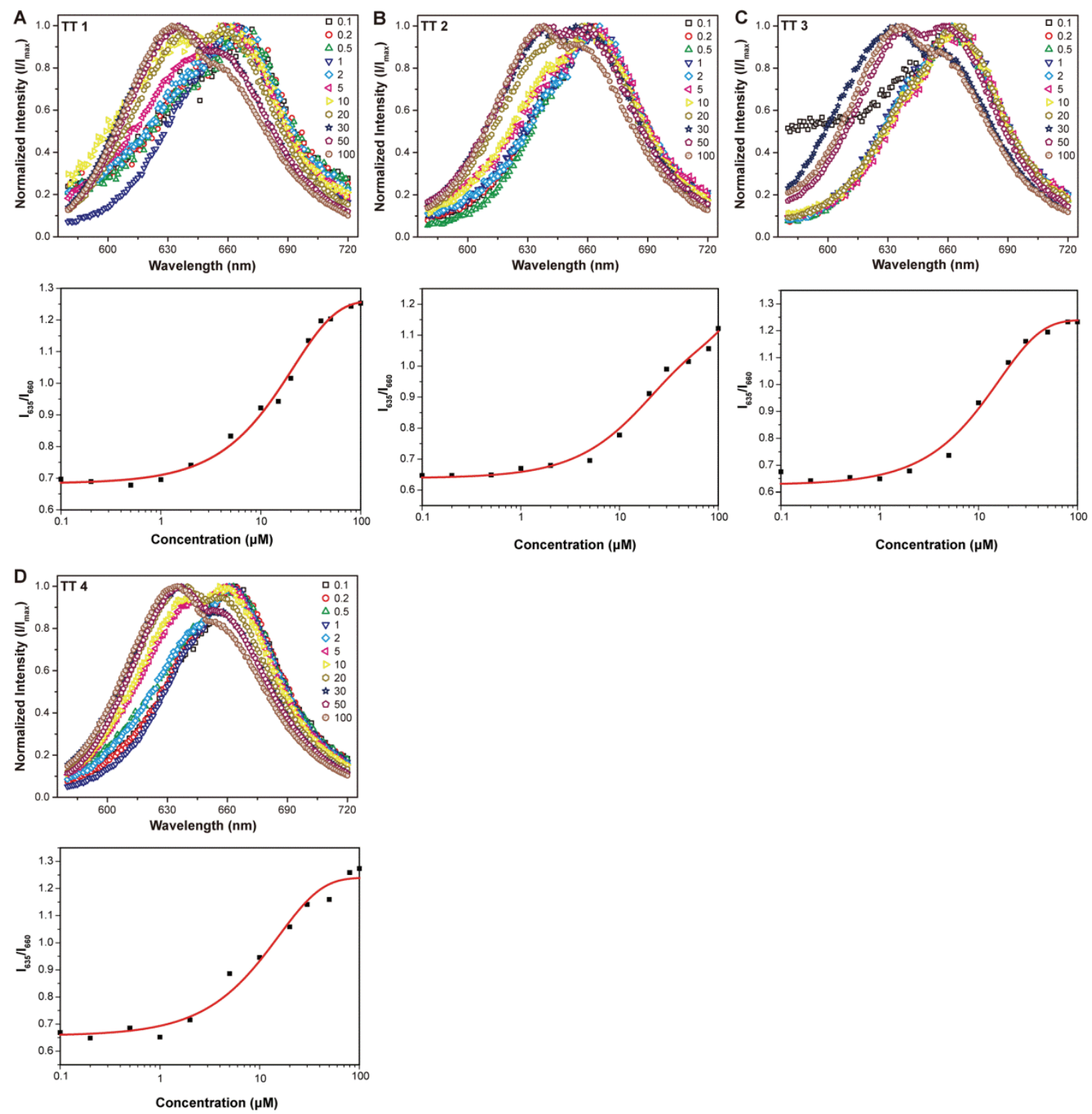

Fig. S7. Critical micellization concentrations (CMC) of the TTs were measured by encapsulation of Nile Red. CMC values of the TTs are within the range of 2-5 $\mathrm{MM}$ regardless of the hydrophilic segment, confirming again the dominant role of the CPT units in stabilizing their supramolecular assemblies. The mechanism of Nile Red encapsulation method is that the Nile Red dye fluoresces intensely in hydrophobic environments (encapsulated) and is strongly quenched and red-shifted in aqueous media (unencapsulated). Plotting the ratio of intensity at $635 \mathrm{~nm}$ (emission maximum of the encapsulated dye) to that at $660 \mathrm{~nm}$ (emission maximum in aqueous conditions) against the concentration of TTs shows the transition that occurs when the concentration of TT monomers exceeds the CMC 

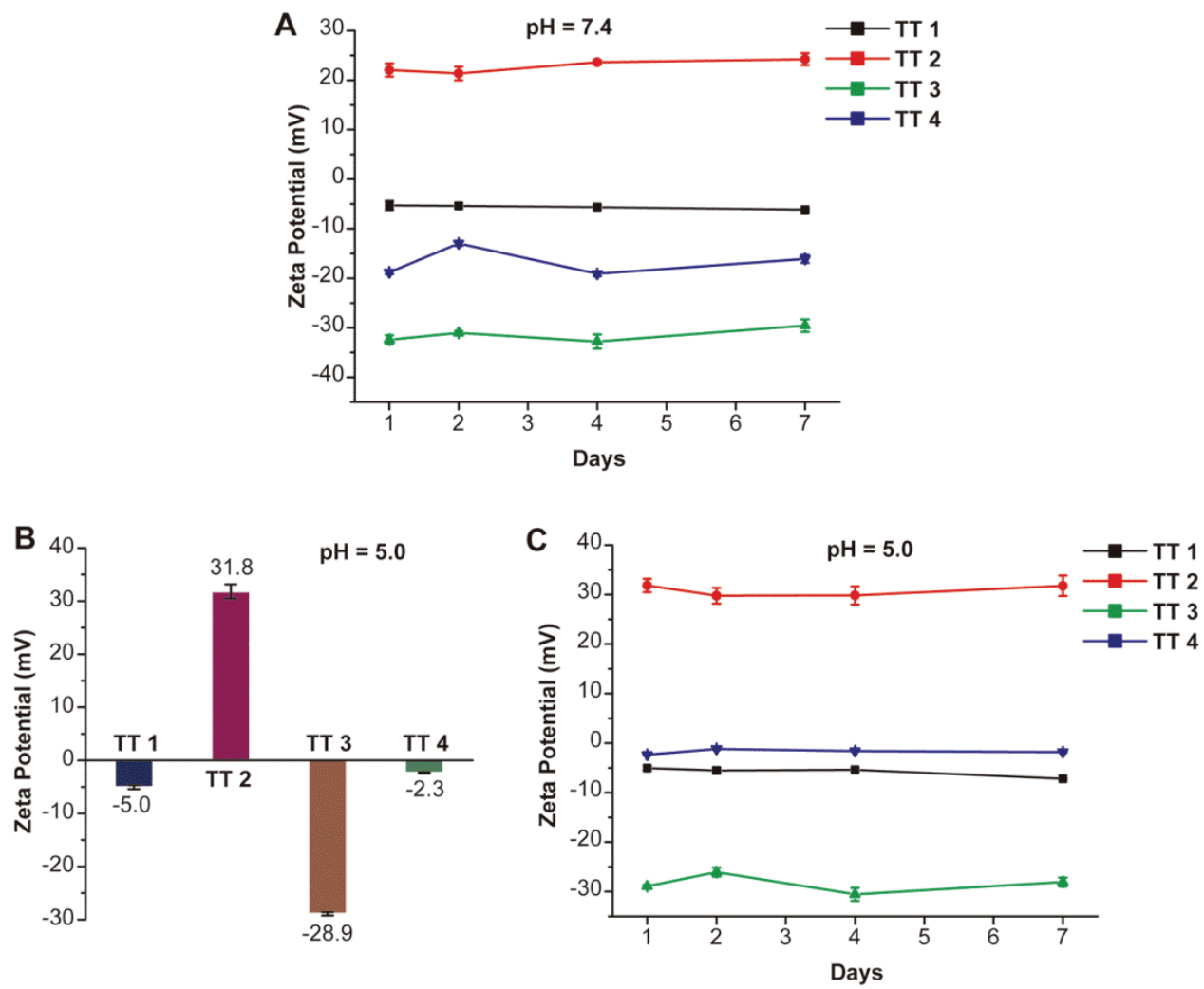

Fig. S8. Variation of $\zeta$-Potential values of self-assembled TT 1-4 in 1X-DPBS buffer at pH 7.4 over time (days 1, 2, 4 and 7) (A); $\zeta$-Potential values of self-assembled TT 1-4 in $1 \mathrm{X}$-DPBS buffer at $\mathrm{pH} 5.0$ (B) and their variation over time (C); The average values and their standard deviations are calculated from three measurements. $\zeta$-Potential values of self-assembled TT 1-4 in 1X-DPBS buffer at pH 7.4 is shown in Fig. 4C. As expected, TT 2 tubular SPs carrying two lysine residues showed a positive value of $22.1 \mathrm{mV}$ at $\mathrm{pH}$ 7.4 and $31.8 \mathrm{mV}$ at $\mathrm{pH} 5.0$ due to the increased protonation of lysine amines. TT 3 showed more negative $\zeta$-potentials at $\mathrm{pH} 7.4$ than 5.0 due to the incorporation of multiple carboxylic groups. The zwitterionic TT 4 tubules carry a more negative charge at $\mathrm{pH} 7.4$ $(-18.8 \mathrm{mV})$ than $\mathrm{pH} 5.0(-2.3 \mathrm{mV})$, likely due to the placement of glutamic acid at the $C$-terminus. In contrast, the non-ionic TT 1 tubular SPs are resistant to the changes in solution $\mathrm{pH}$, having a consistent negative value of $-5 \mathrm{mV}$ at both $\mathrm{pH} 7.4$ and 5.0. In addition, the $\zeta$-potential values of TTs are stable over seven days, indicating their long-term stability. 


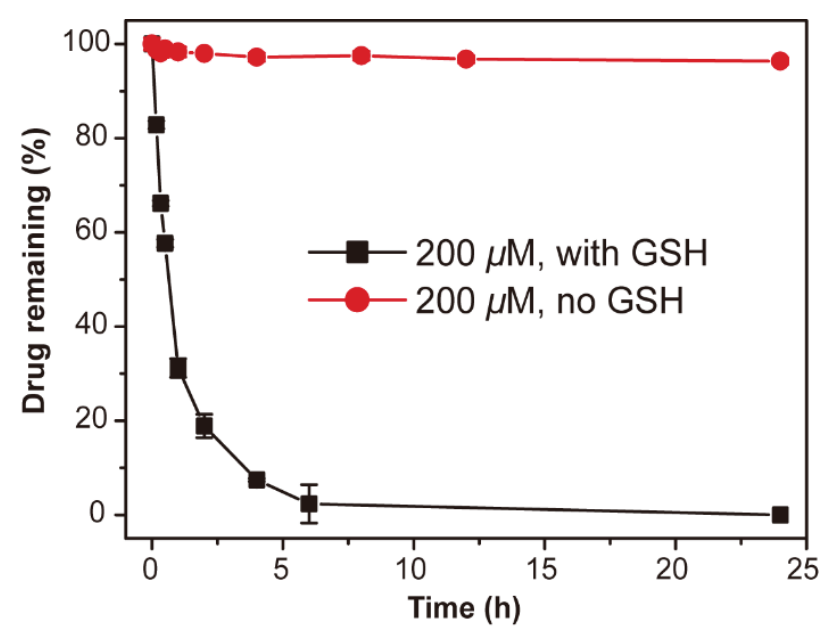

Fig. S9. Drug release plot of TT 1 at a concentration of $200 \mu \mathrm{M}$ with or without $10 \mathrm{mM}$ GSH in buffer. $80 \%$ of the conjugated CPT molecules were released within $2 \mathrm{~h}$ in the presence of $10 \mathrm{mM} \mathrm{GSH}$, reaching almost $100 \%$ by $6 \mathrm{~h}$, while only a slight amount (less than $10 \%$ ) of the conjugates had degraded (by hydrolysis) in $24 \mathrm{~h}$ without GSH. These results indicate that the therapeutic supramolecular polymers are able to undergo bioconversion to the parent drug and exert the pharmaceutical and biological functionalities of monomeric CPTs.

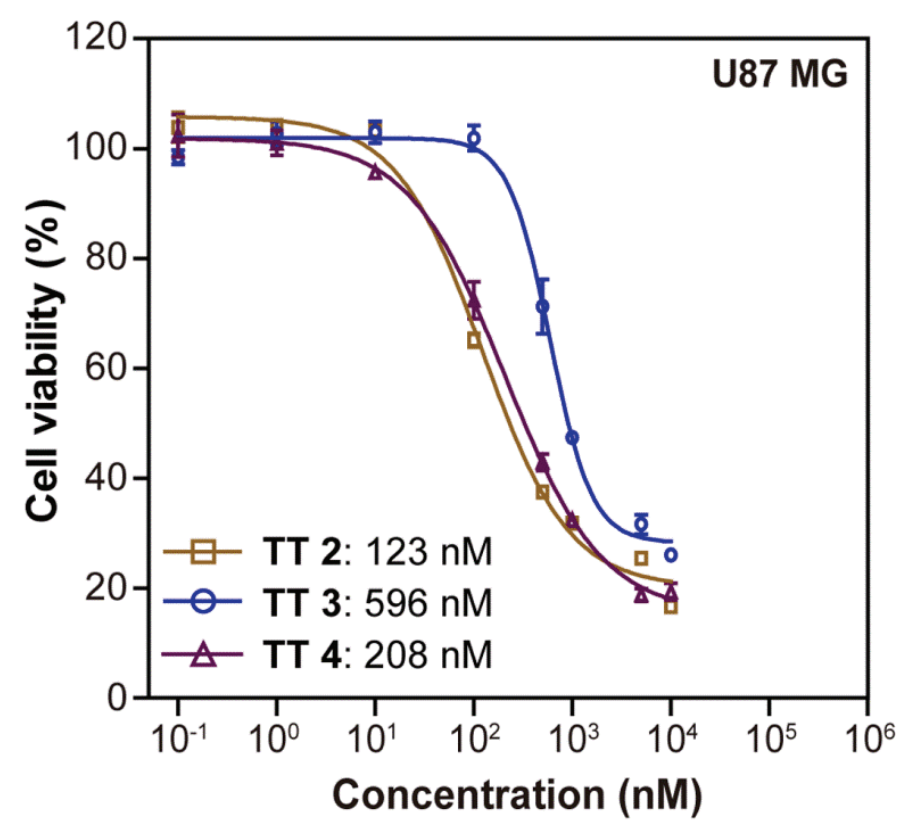

Fig. S10. In vitro toxicity of TT $2-4$ against the U87 MG brain cancer cell line, with both free CPT and Irinotecan as controls (48 $\mathrm{h}$ incubation). 


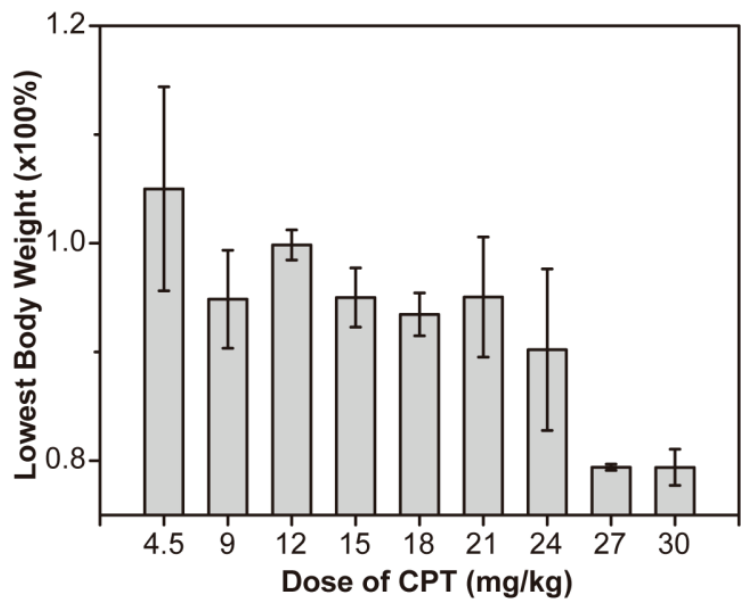

Fig. S11. Maximum tolerated dose (MTD) study showing the averaged lowest body weight recorded per corresponding dosage of TT 1 ( $n=3$ for each dosage group). MTD was determined by intravenously administering TT 1 in a dose escalation study in healthy athymic nude mice. The MTD of TT 1 is around $24 \mathrm{mg} / \mathrm{kg}$ (CPT equivalent), which greatly exceeds MTD of free CPT $(\sim 5 \mathrm{mg} / \mathrm{kg})$. The maximum tolerated dose (MTD) was defined by the largest dose that did not result in more than a $20 \%$ mean body weight loss or death of an animal in that group. Doses of 54 and $36 \mathrm{mg} / \mathrm{kg}$ caused at least one death in each group.
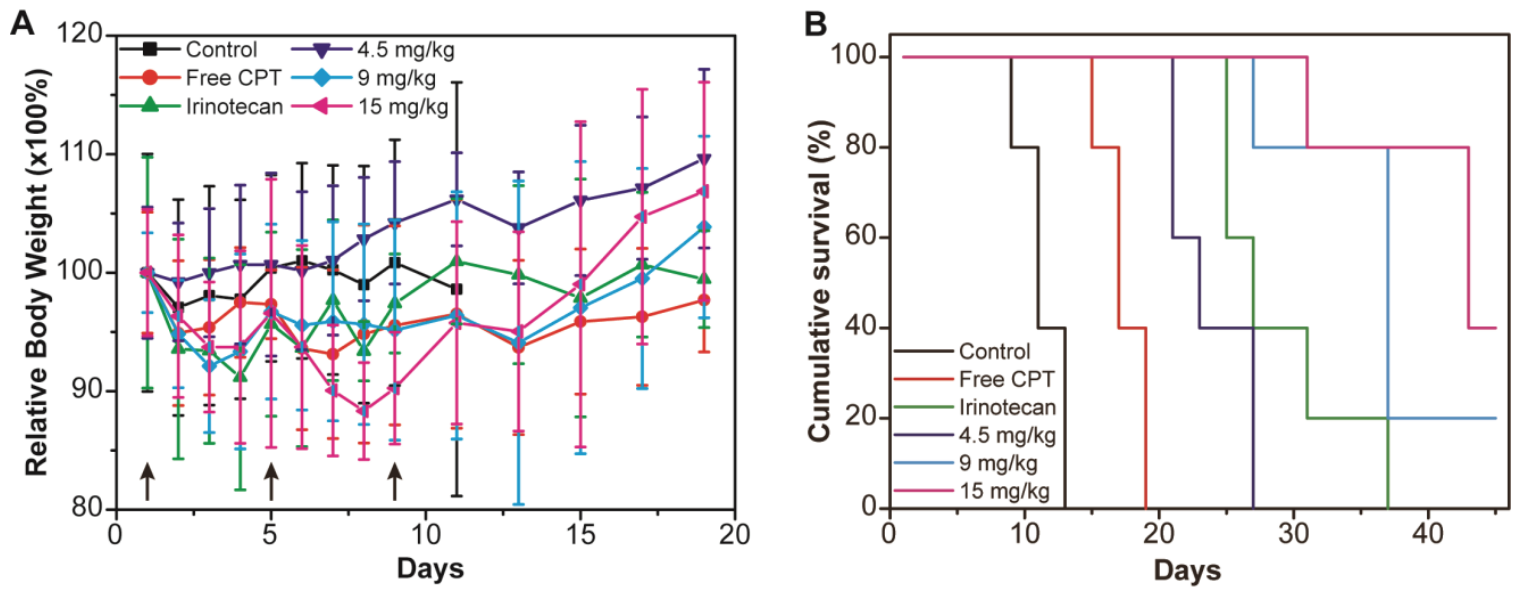

Fig. S12. Body weight change of mice (A) and cumulative survival plot of mice (B) in systemic delivery of TT 1. Groups treated with drugs (except the $4.5 \mathrm{mg} / \mathrm{kg}$ group) showed slight average body weight decreases (less than 10\%), however, they were all within the acceptable toxicity range. We consider $20 \%$ body weight loss as significantly toxic. Much improved median survivals of mice were observed for TT 1 at $9 \mathrm{mg} / \mathrm{kg}$ (37 d) and $15 \mathrm{mg} / \mathrm{kg}$ (43 d) respectively, compared with control (11 d), free CPT (17 d), TT 1 at $4.5 \mathrm{mg} / \mathrm{kg}(23 \mathrm{~d})$, and irinotecan (27 d). 


\begin{tabular}{|c|c|}
\hline Self-assembled Tubustecans & Diameters measured by conventional TEM \\
\hline TT 1 & $8.8 \pm 0.8 \mathrm{~nm}$ \\
\hline TT 2 & $8.1 \pm 0.6 \mathrm{~nm}$ \\
\hline TT 3 & $8.4 \pm 0.9 \mathrm{~nm}$ \\
\hline TT 4 & $8.3 \pm 0.9 \mathrm{~nm}$ \\
\hline
\end{tabular}

Table S1. Diameters of self-assembled TT tubular supramolecular polymers (SPs) measured from conventional-TEM $(n>40)$. The lengths of these functional SPs are all on the micrometer scale and the diameters are $8.8 \mathrm{~nm}$ for TT 1 and in the range of 8.1-8.4 $\mathrm{nm}$ for TT 2-4, as measured from TEM images. The diameters of TT 2-4 are slightly smaller $(\sim 0.5 \mathrm{~nm})$ than TT 1 because OEGs on the lysine side chain extend the molecular length of TT 1. The measured diameters strongly support the monolayered packing model for these tubular SPs. 


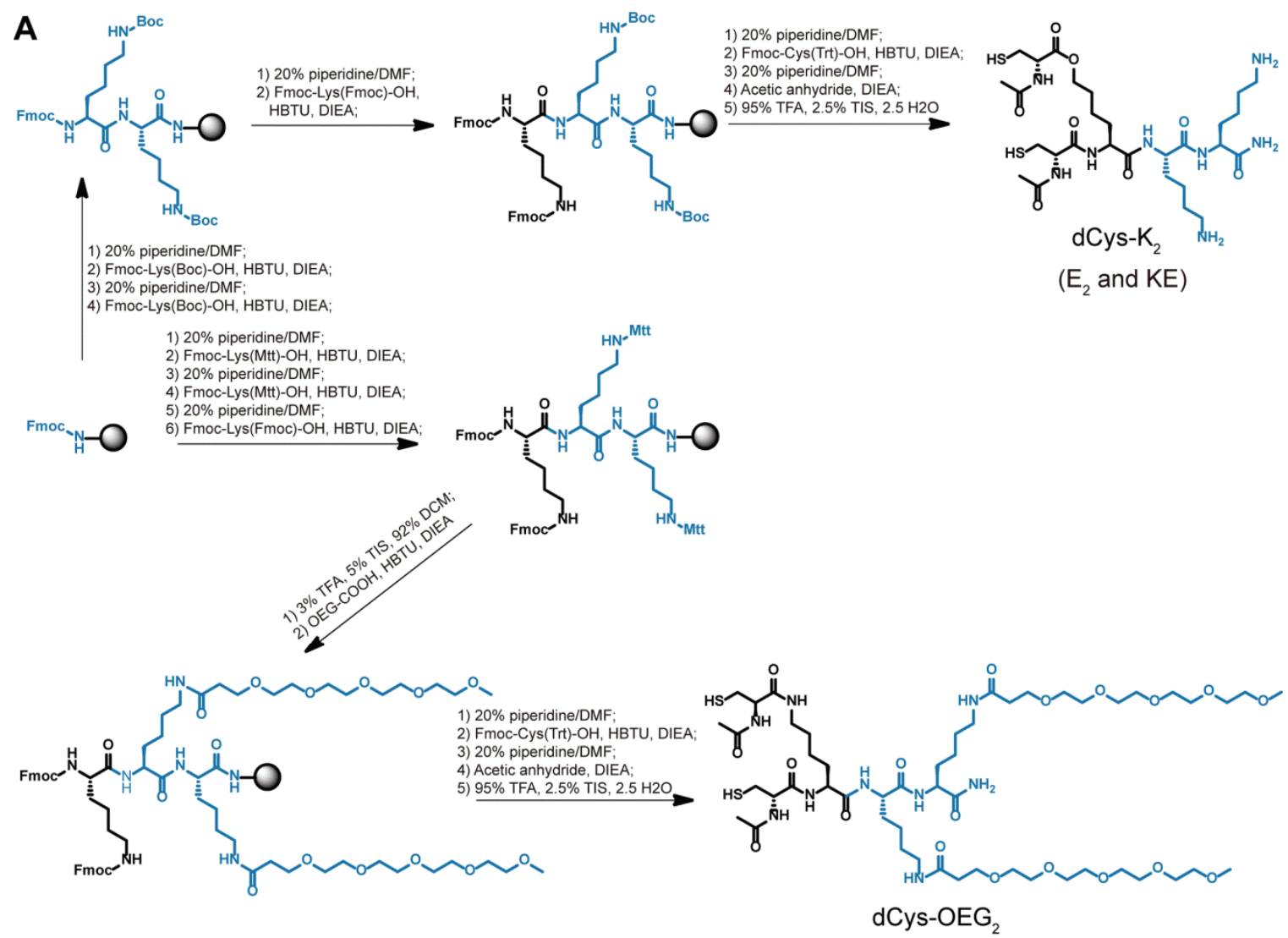

B

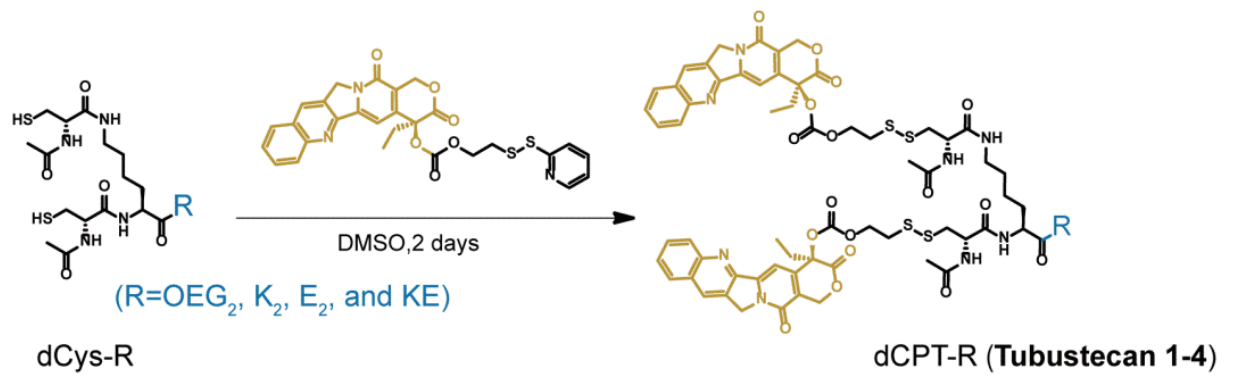

Scheme S1. Schematic illustration for synthesis of Tubustecans (TTs). (A) Synthetic routes to peptide segments $\mathrm{dCys}_{2}-\mathrm{K}_{2}$ and $\mathrm{dCys}_{-} \mathrm{OEG}_{2}$ using standard Fmoc solid phase peptide techniques (dCys- $\mathrm{E}_{2}$ and $\mathrm{dCys}-\mathrm{KE}$ use similar protocols to $\mathrm{dCys}_{2}-\mathrm{K}_{2}$ ). (B) Synthesis of functional TT 1-4 by mixing peptide segments synthesized in (A) with CPT-etcSS-Pyr in DMSO. 

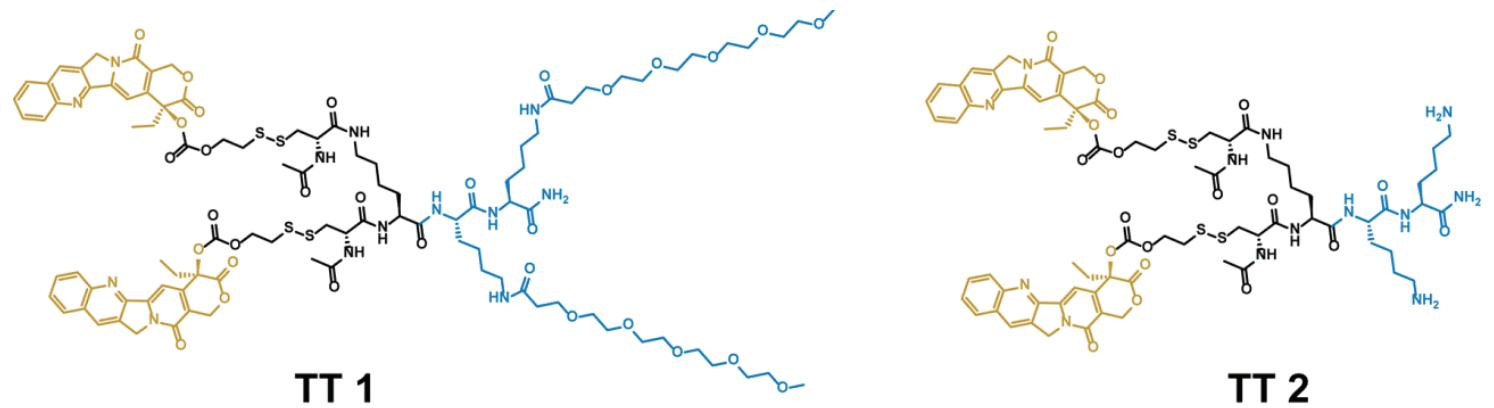

TT 2

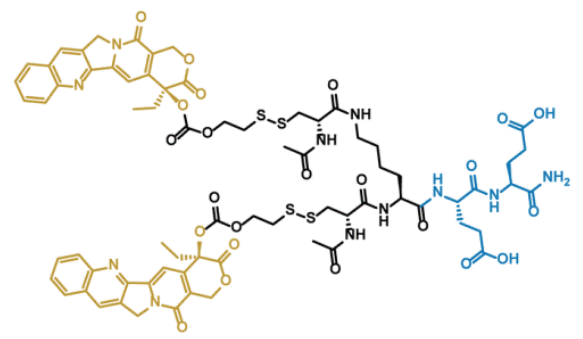

TT 3

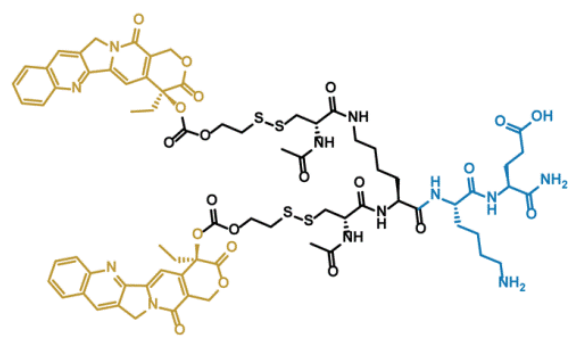

TT 4

Scheme S2. Chemical structures of TT 1-4.<smiles>CCN(CC)c1ccc2cc(-c3nc4ccccc4s3)c(=O)oc2c1</smiles>

Coumarin 6<smiles>O=C1OC2(c3cc(I)c(O)c(I)c3Oc3c2cc(I)c(O)c3I)c2c(Cl)c(Cl)c(Cl)c(Cl)c21</smiles>

Rose Bengal lactone<smiles>CCN(CC)c1ccc2nc3c4ccccc4c(=O)cc-3oc2c1</smiles>

Nile Red<smiles>CCCN1/C(=C/C=C2\CCCC(/C=C/C3=[N+](CCC)c4ccccc4C3(C)C)=C2Cl)C(C)(C)c2ccccc21</smiles>

IR 780 iodide

Scheme S3. Chemical structures of the four different dyes encapsulated within TT 1. 